\title{
Behavioral and Neurochemical Alterations Following Thiamine Deficiency in Rodents: Relationship to Functions of Cholinergic Neurons
}

\author{
Osamu NAKAGAWASAI \\ Department of Pharmacology, Tohoku Pharmaceutical University, \\ 4-4-1 Komatsushima, Aoba-ku, Sendai 981-8558, Japan
}

(Received April 13, 2005)

\begin{abstract}
Memory deficits are induced during the late stage (20-25 days) of thiamine-deficient (TD) feeding. In this review, the role of cholinergic neurons on the memory deficit induced by TD feeding are summarized. Although memory deficit cannot be suppressed by an injection of thiamine once it appears, such impairment was found to be protected by early treatment with thiamine during TD feeding. Administration of muscarinic $\mathrm{M}_{1}$ agonist McN-A-343 reversed the memory deficit observed in TD mice, although the muscarinic $\mathrm{M}_{2}$ antagonist methoctramine did not. The "kampo" (traditional herbal) medicine, "kami-untan-to" (KUT), protected against the memory deficit observed in TD mice. Choline acetyltransferase (ChAT) fluorescence intensity, a marker of presynapse of cholinergic neurons, was decreased in the cortex and hippocampus at an early stage (14th day) of TD, and it was decreased in a wide range of brain areas at a late stage (25th day) of TD. Early KUT treatment inhibited the reduction of ChAT in the hippocampus of TD mice. These findings suggested that the memory deficit may be caused by a reduction in the cholinergic function at an early stage of $\mathrm{TD}$, and that the activation of cholinergic neurons may play an important role in the improvement of TD-induced memory deficit.
\end{abstract}

Key words — thiamine deficiency; memory; choline acetyltransferase; kami-untan-to; muscarinic receptors

\section{INTRODUCTION}

Thiamine is present extracellularly as unphosphorylated thiamine and thiamine monophosphate, and intracellularly, it is often thiamine pyrophosphate (the physiologically active form of thiamine). Thiamine pyrophosphate acts as a coenzyme for various enzymes, such as transketolase, pyruvate dehydrogenase and $\alpha$-ketoglutarate dehydrogenase in glucose metabolism (Fig. 1). Unphosphorylated thiamine has been proposed to act as a shuttle molecule across cell membranes, ${ }^{1}$ and it may also play a part in nerve conduction and synapses. ${ }^{2)}$ Thiamine triphosphate appears to play an important role in membrane conductance. ${ }^{3)}$ Furthermore, it is known that thiamine is involved in the syntheses of acetylcholine, $\gamma$-aminobutyrate, glutamate and asparate.

Thiamine deficiency (TD) leads to conditions such as beriberi and Wernicke-Korsakoff's syndrome (WKS), which is characterized by selective cell loss, ${ }^{4)}$ cholinergic deficits ${ }^{5,6)}$ and cognitive deficits. ${ }^{7)}$ These changes also occur in Alzheimer's disease (AD) .8,9) Treatment with high doses of parenteral thiamine

This review is commemorated for Award of PSJ Tohoku Branch to Excellent Scientists, 2004.

e-mail: osamun@tohoku-pharm.ac.jp generally leads to the prompt clinical improvement of beriberi and Wernicke's encephalopathy. It is also reported that the progress of dementia in AD patients can be halted by a timely injection of a bolus dose of thiamine. ${ }^{10)}$ Lack of appropriate treatment is often lethal, and delayed treatment is followed by subsequent serious symptoms. ${ }^{7)}$ Therefore, it is imperative to precisely elucidate the mechanism of the abnormal behaviors induced by TD.

There are two types of experimental models of TD: antithiamine drug (pyrithiamine) - and dietary-induced TD. In this review article, we propose the role of the cholinergic systems in the brain in memory deficit in dietary-induced TD, based on our findings of behavioral testing and immunohistochemical analysis.

\section{ABNORMAL BEHAVIORS INDUCED BY THIAMINE DEFICIENCY}

Dietary-induced TD causes various abnormal behaviors in rats and mice (Table 1). Escape behavior and increased startle response appear during the early stage of a TD diet. ${ }^{11,12)}$ After 12-14 days of TD treatment, bradycardia, ${ }^{13)}$ lack of appetite ${ }^{14)}$ and hypothermia ${ }^{15,16)}$ appear. Persistent erection, ${ }^{17)}$ hypotension, ${ }^{13)}$ increased pain threshold, ${ }^{18-20)}$ muri- 


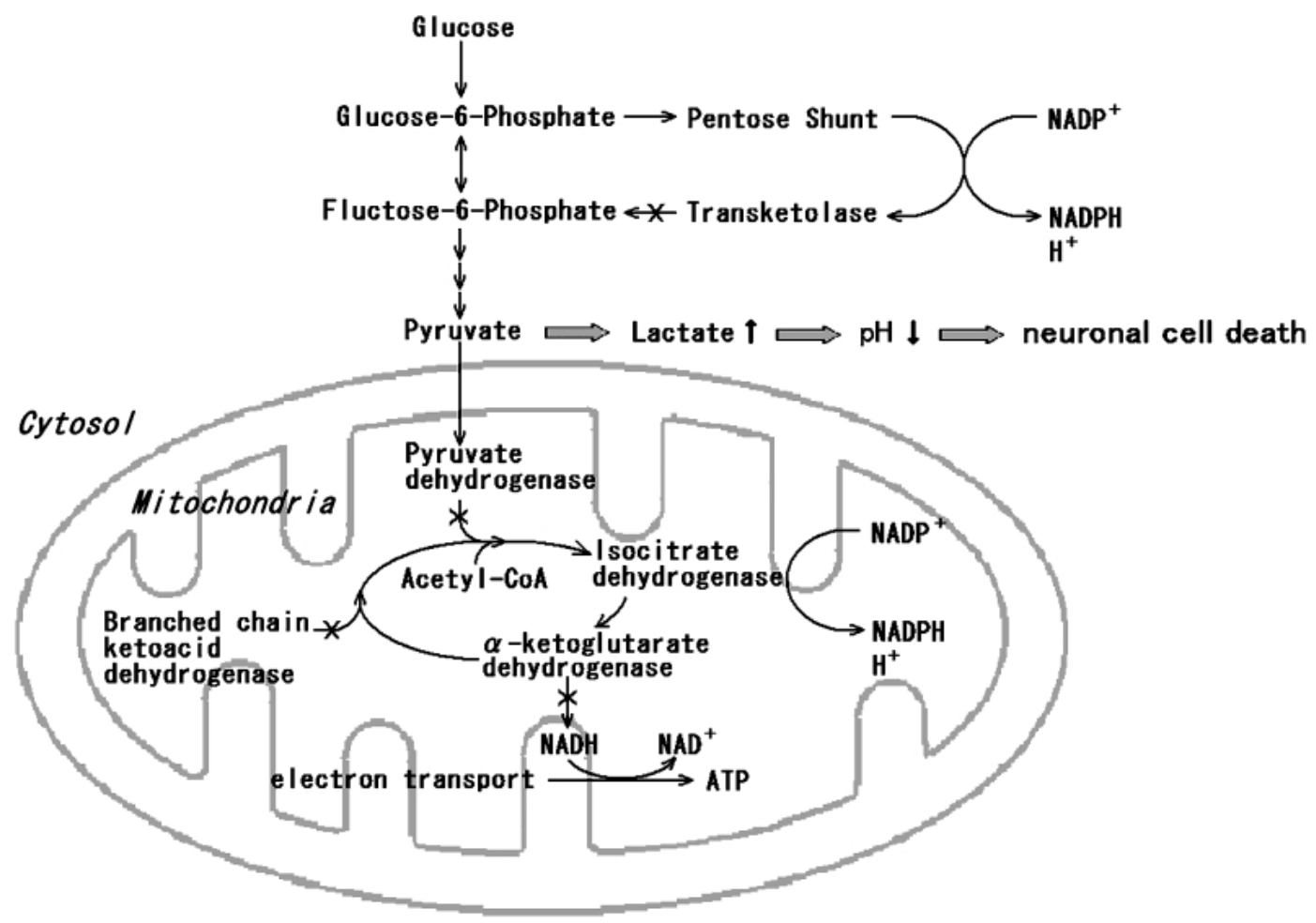

Fig. 1. Mechanism of Neuronal Cell Death Due to Thiamine Deficiency in Glucose Metabolism $\mathrm{X}$ : enzyme activity is reduced by TD.

Table 1. Abnormal Behaviors Induced by Thiamine Deficiency

\begin{tabular}{ll}
\hline \hline \multicolumn{1}{c}{ Behaviors } & \multicolumn{1}{c}{ References } \\
\hline Increased startle response & Peskin et al., 1967(11); Onodera et al., 1978 (12) \\
Bradycardia & Onodera et al., 1991 (13) \\
Lack of appetite & Onodera et al., 1981 (14) \\
Hypothermia & Lee et al., 1985 (15); Onodera et al., 1990 (16) \\
Persistent erection & Onodera et al., 1978 (17) \\
Hypotension & Onodera et al., 1991 (13) \\
Increased muricidal behavior & Onodera et al., 1978 (12); Tadano et al., 1997 (21) \\
Increased pain threshold & Tadano et al., 1995 (18) and 1999 (19); Nakagawasai et al., 2001 (20) \\
Learning and memory deficits & Mair et al., 1991 (33, 34); Langlais et al., 1992 (31) and 1995 (32); \\
& Nakagawasai et al., 2000 (22, 23) and 2001 (24) \\
Depressive behavior & Nakagawasai et al., 2001 (25) \\
Altered circadian rhythm & Bennett et al., 1999 (26) \\
\hline
\end{tabular}

cide (mouse-killing behavior), ${ }^{12,21)}$ impairment of memory, ${ }^{22-24)}$ increased immobility time in the forced swimming test ${ }^{25}$ ) and altered circadian rhythm $\left.{ }^{26}\right)$ are observed during the late stage (20-30 days) of a TD diet. Among the above-mentioned behaviors, muricide, increased pain threshold, memory deficit and increased immobility time, once they appear, cannot be suppressed by an injection of thiamine $\mathrm{HCl}$. These findings indicate that these responses may result from irreversible changes in cer- tain brain and/or spinal regions caused by TD.

Muricide and increased immobility time in the forced swimming test have been regarded as a model of depression, since these responses are markedly suppressed by antidepressant drugs. ${ }^{14,27)}$ The incidence of muricide in rats is reported to be $5 \%$ on the 14 th day and $60 \%$ on the $20-25$ th day after the start of TD feeding. ${ }^{12)}$ Regarding pharmacological effects, muricide is suppressed by 5-hydroxytryptophan, a serotonin precursor, ${ }^{28)}$ chlorimipramine, a serotonin reup- 
take inhibitor, ${ }^{14)}$ L-threo-dihydroxyphenylserine, a direct precursor of norepinephrine ${ }^{29)}$ and desmethylimipramine, a norepinephrine reuptake inhibitor. ${ }^{14)}$ Furthermore, increased immobility time is decreased by imipramine, a serotonin and norepinephrine reuptake inhibitor. ${ }^{25)}$ These results indicate that a developmental mechanism of TD diet-induced muricide and increased immobility time may be involved in the degeneration of the central serotonergic and noradrenergic neurons.

The increased pain threshold in TD rats has been recognized by hot-plate test, the tail-flick test ${ }^{18,19)}$ and the formalin test ${ }^{20)}$ in the late stage of a TD diet. We have previously examined the correlation between changes in substance P (SP) and somatostatin (SST) distribution in the rat spinal cord and increased pain threshold during TD feeding, as determined by our immunohistochemical analysis. The SP and SST contents were significantly decreased in the dorsal spinal cord. ${ }^{18,19)}$ The behavioral responses to SP and SST injected intrathecally were enhanced in TD mice. ${ }^{20)}$ Thus, increased pain threshold induced by TD may involve, at least in part, irreversibly damaged SP and SST neurons in TD animals, since TD produces an enhanced sensitivity of SP and SST in the spinal cord after the development of irreversible neuronal lesions. ${ }^{20)}$

One of mechanisms of the neuronal cell death in TD is well known (Fig. 1). Thiamine plays an important role in glucose metabolism. Thus, TD leads to a decrease in media glucose levels which is consistent with the increased glucose utilization and is reflected in the observed increase in lactate production. Consistent with the rise of lactate in cells, extracellular $\mathrm{pH}$ is decreased. It has been proposed that lactic acidosis could be the cause of neuronal cell death in TD rodents. ${ }^{30)}$

\section{MEMORY DEFICIT INDUCED BY THIAMINE DEFICIENCY}

Memory deficit as determined by the passiveavoidance task is observed on days $20-25$ in dietaryinduced TD rats and mice. ${ }^{22-24)}$ Memory deficit due to antithiamine-induced TD has also been demonstrated by the water maze task, ${ }^{31)}$ T-maze task, ${ }^{32)}$ a nonmatching-to-sample task, ${ }^{33)}$ and matching-tosample task. ${ }^{34)}$ These results suggest that TD induces a working memory impairment in rodents. This type of working memory impairment has been seen in aged humans ${ }^{35)}$ and in WKS patients. ${ }^{36)}$ We have investigated the effects of cholinergic drugs on memory deficit in rats and mice after TD feeding. Memory deficit was improved by the administration of chronic physostigmine [intraperitoneally (i.p.)], a cholinesterase inhibitor, ${ }^{23)}$ and oxotremorine (i.p.), a nonselective muscarinic agonist. ${ }^{24)}$ However, administration of lobeline (i.p.), a nicotinic agonist, did not reverse memory deficit. ${ }^{24)}$ Another group has also reported that in antithiamine-induced TD animals the performance in the string test (standardized test of neurological competence that measures ability to traverse a string) was reversed by the injection of physostigmine and arecholine, muscarinic agonists, but not by nicotine. ${ }^{5)}$ Moreover, we examined the role of muscarinic receptor subtypes on the memory deficit induced by TD feeding. Stimulation of postsynaptic muscarinic $M_{1}$ and inhibition of presynaptic $M_{2}$ receptors can enhance the acquisition of spatial reference memory in both the water maze test and the nonmatching-to-position test. ${ }^{37)}$ Intracerebroventricular (i.c.v.) administration of $\mathrm{McN}-\mathrm{A}-343$, a muscarinic $\mathrm{M}_{1}$-receptor agonist, improved the memory deficit in TD mice. Interestingly, methoctramine (i.c.v.), a selective muscarinic $\mathrm{M}_{2}$-receptor antagonist, did not have a significant effect on TD-induced memory deficits. ${ }^{24)}$ It has been demonstrated that the intensity of choline acetyltransferase (ChAT) fluorescence, a marker of presynapse of cholinergic neurons, is decreased in the cortex, hippocampus and thalamus in the late stage in TD rats. These findings suggest that methoctramine does not have a positive effect on the impairment of memory function since the presynaptic cholinergic neurons were degenerated after a TD diet. Thus, the stimulation of muscarinic $\mathrm{M}_{1}$ receptors plays an important role in the improvement of memory deficit induced by TD feeding.

\section{EFFECT OF KAMI-UNTAN-TO ON MEMORY DEFICITS INDUCED BY THIAMINE DEFICIENCY}

The "kampo" medicine "kami-untan-to" (KUT; Jia-wei-wen-dan-tang in Chinese), which was first described in the Japanese literature in 1626, has been used for a long time in the treatment of neuropsychiatric problems such as neurosis or insomnia. Oral administration of KUT is reported to improve the cognitive function in aged rats and basal forebrainlesioned rats, via an increased ChAT activity in the 
brain. ${ }^{38,39)}$ Loss of magnocellular basal forebrain has been found in WKS patients ${ }^{40}$ ) and in the pyrithiamine-induced TD model. ${ }^{41)}$ Our colleagues observed that the memory disturbance improved in AD patients who took KUT for about 1 year. ${ }^{42)}$ In our study, daily administration of KUT from the 1st day of TD feeding prevented the memory deficits. ${ }^{43)}$ Thiamine comprised $32 \mathrm{ng} / \mathrm{ml}$ of KUT. First, we examined the effect of water containing $32 \mathrm{ng}$ thiamine/ $\mathrm{ml}$ on physiological changes and the memory-related behavior after TD feeding. There was no influence of thiamine within the KUT-administered group in this investigation compared with the TD group. It may be accordingly considered that the protection effect of memory-related behavior may have lead to the effect of KUT itself. Furthermore, with respect to the memory improvement effect of KUT, the following reports should be considered. Oral administration of KUT is reported to increase the latency time in ibotenic acid-induced basal forebrain-lesioned rats ${ }^{38)}$ and in aged rats $^{39)}$ in the step-through passive-avoidance task, via an increased ChAT activity in the frontoparietal cortex. Thus, the possibility exists that the effect of KUT may be due to influences on the cholinergic neuronal system in TD mice.

It has been indicated that thiamine-dependent enzymes and ChAT activity are decreased in $\mathrm{AD}$ patients ${ }^{44,45}$ and in WKS patients. These enzymes are closely related to the synthesis of ACh. We have already shown that the ChAT fluorescence intensity in the brain is decreased in TD rats and this decrease is correlated with the impaired memory ability as in WKS. ${ }^{23)}$ Moreover, pyrithiamine-induced TD rats display a reduction in hippocampal $\mathrm{ACh}$ release during spatial memory performance. ${ }^{46)}$ Fadda et al. ${ }^{47)}$ demonstrated that the magnitude of ACh output in the hippocampus is positively correlated with spatial memory behavior in freely moving rats. Since TD induces the impairment of spatial memory using the elevated plus-maze task, we measured the ChAT fluorescence intensity in the fields of CA1, CA2, CA3 and DG in the hippocampus. In the TD group, the fluorescence intensity of ChAT showed a marked decrease in the entire region of the hippocampus on the 20th day of TD feeding. As the impaired memoryrelated behavior induced by TD was protected by chronic KUT treatment from the 1st day, KUT may be able to delay the impairment of the cholinergic system in the hippocampus. Actually, the ChAT fluores- cence intensity of KUT-treated TD mice remained increased in comparison with non-treated TD mice. Moreover, the ChAT level was restored in CA1, CA2 and CA3 of the hippocampus by KUT treatment in comparison with the PF group. The protection of ChAT induced by KUT treatment from the 1st day was markedly high in CA1 and CA 3 fields. It has been demonstrated that oral administration of KUT enhances the ACh levels in the striatum, hippocampus and cortex, but the choline levels are not increased in any brain region. ${ }^{48)}$ In addition, oral administration of KUT increased ChAT mRNA in the basal forebrain and nerve growth factor (NGF) mRNA in the frontopanetal cortex in aged rats. NGF has been shown to increase ChAT levels, induction of NGF has generally been considered to be an essential step of ChAT induction. These findings suggest that the positive effect of KUT on memory-related behavior in TD mice might result from an increased level of synthesis of ACh by induction of ChAT in the hippocampus. However, it is still unclear which herb among 13 kinds of crude drug components plays the critically essential part in the potentiation of neurons. Another group has already reported that Polygalae Radix, one of the components of KUT increases the ChAT activity in rat basal forebrain cell culture. ${ }^{49)}$ Nevertheless, other herbs or the interaction of some herbs with the Polygalae Radix should be considered. In order to clarify the detailed mechanism of KUT on the cholinergic system and the active ingredients which induce the neurotrophic-like effect, we are currently undertaking further experiments. Our findings suggests that KUT treatment may prevent the memory deficit induced by TD feeding via protection against the decrease of ChAT in the hippocampus of TD mice. KUT may be useful as a potential remedy in the treatment of patients with WKS.

\section{CONCLUSIONS}

In consideration of the above findings and review, the memory deficit induced by TD may be considered to be caused by degeneration of cholinergic neurons, and the activation of cholinergic neurons may play an important role in the improvement of TD-induced amnesia.

Antithiamine- and dietary-induced TD animals are useful experimental models of WKS. KUT may be useful as a potential remedy in the treatment of patients with WKS (Fig. 2). 


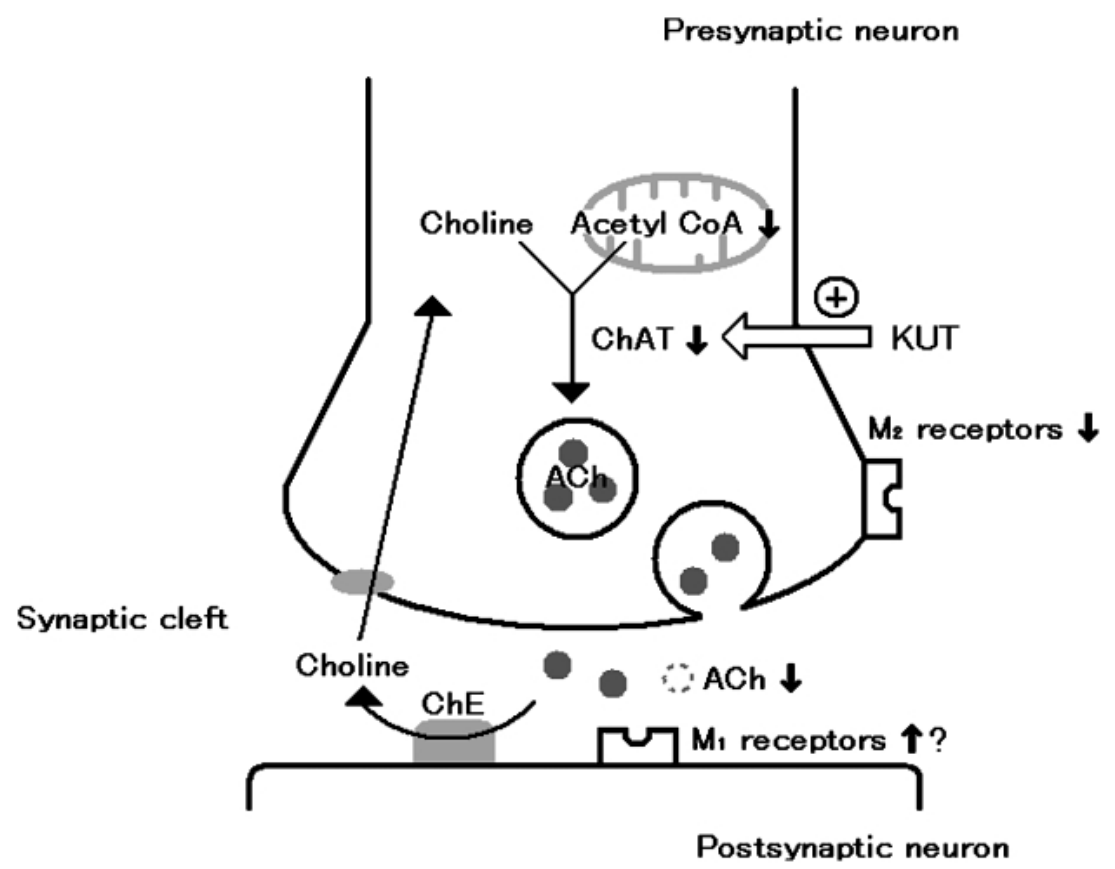

Fig. 2. Schematic Illustration of the Hypothetical Mechanisms on Thiamine Deficiency (TD) -induced Memory Impairment in the Hippocampus

TD lead to a decreased acetyl CoA level. The reduced ChAT and $\mathrm{M}_{2}$ receptor level in presynaptic cholinergic neurons could be due to the irreversible neurodegeneration caused by TD. Thus, this degeneration seems to be compensated for by an upregulation of postsynaptic $\mathrm{M}_{1}$ receptors. KUT might cause ChAT induction in the hippocampus.

Acknowledgements I would like to express deep gratitude to my collaborators for their kind help, support and valuable suggestion in this study: the late Prof. Kensuke Kisara, Prof. Takeshi Tadano, Associate Prof. Koichi Tan-No, Ms. Fukie Niijima, Ms. Miyuki Namioka, Mr. Soichi Hozumi, Mr. Ryoo Taniguchi, Mr. Fumihiro Yamadera, Mr. Atsunobu Murata and Ms. Shoko Sato, Tohoku Pharmaceutical University and Associate Prof. Yuichiro Arai, Showa University. This study was supported in part by Grants-in-Aid from the Ministry of Education, Science, Culture and Sports of Japan (08672622, 13771442 and 16790232).

\section{REFERENCES}

1) Brown R. D., J. Theor. Biol., 143, 565-573 (1990) .

2) Cooper J. R., Pincus J. H., Neurochem. Res., 4, 23-29 (1979).

3) Bettendorff L., Wins P., Recent Res. Dev. Neurochem., 2, 37-62 (1999).

4) Langlais P. J., Mair R. G., J. Neurosci., 10, 1664-1674 (1990).

5) Barclay L. L., Gibson G. E., Blass J. P., J. Pharm. Exp. Ther., 217, 537-543 (1981).

6) Barclay L. L., Gibson G. E., Blass J. P., J.
Nutr., 112, 1906-1913 (1982).

7) Victor M., Adams R. D., Collins G. H., "The Wernicke-Korsakoff Syndrome and Related Neurologic Disorders due to Alcoholis," F. A. Davis Company, Philadelphia, 1989.

8) Arendt T., Bigl V., Arendt A., Tennstedt A., Acta Neuropathol., 61, 101-108 (1983).

9) Whitehouse P. J., Price D. L., Clark A. W., Coyle J. T., DeLong M. R., Ann. Neurol., 10, 122-126 (1981).

10) Mimori Y., Katsuoka H., Nakamura S., Metab. Brain Dis., 11, 89-94 (1996) .

11) Peskin M. R., Newton G., Brin M., J. Nutr., 91, 20-24 (1967).

12) Onodera K., Tadano T., Sakai K., Kisara K., Ogura Y., Folia Pharmacol. Japon., 74, 641648 (1978).

13) Onodera K., Saito T., Ito M., Jpn. J. Psychopharmacol., 11, 319-325 (1991).

14) Onodera K., Ogura Y., Kisara K., Physiol. Behav., 27, 847-853 (1981).

15) Lee R. S., Strahlendorf H. K., Strahlendorf J. C., Brain Res., 327, 249-258 (1985).

16) Onodera K., Shinoda H., Watanabe T., Jpn. J. Pharmacol., 54, 339-343 (1990).

17) Onodera K., Tadano T., Kisara K., Kimura 
Y., Ogura Y., Andrologia, 10, 467-472 (1978).

18) Tadano T., Asao T., Aizawa T., Sakurada S., Abe Y., Yonezawa A., Ando R., Arai Y., Kinemuchi H., Kisara K., Brain Res., 696, 2129 (1995).

19) Tadano T., Asao T., Aizawa T., Nakagawasai O., Tan-No K., Niijima F., Takahashi N., Arai Y., Yasuhara H., Kisara K., Biogen. Amines., 15, 251-262 (1999).

20) Nakagawasai O., Tadano T., Tan-No K., Hozumi S., Taniguchi R., Niijima F., Asao T., Kinemuchi H., Arai Y., Yasuhara H., Kisara K., Life Sci., 69, 1155-1166 (2001).

21) Tadano T., Abe Y., Morikawa Y., Asao T., Hozumi M., Takahashi N., Tan-No K., Kisara K., Meth. Find. Exp. Clin. Pharmacol., 19, 527-531 (1997).

22) Nakagawasai O., Tadano T., Niijima F., TanNo K., Kisara K., Brain Res. Bull., 51, 47-55 (2000)

23) Nakagawasai O., Tadano T., Hozumi S., TanNo K., Niijima F., Kisara K., Brain Res Bull., 52, 189-196 (2000).

24) Nakagawasai O., Tadano T., Hozumi S., Taniguchi R., Yamadera F., Tan-No K., Niijima F., Arai Y., Yasuhara H., Kinemuchi H., Kisara K., Biogen. Amines., 16, 199-210 (2001).

25) Nakagawasai O., Tadano T., Hozumi S., Taniguchi R., Tan-No K., Esashi A., Niijima F., Kisara K., Life Sci., 69, 1181-1191 (2001).

26) Bennett M. R., Schwartz W. J., J. Neurol. Sci., 163, 6-10 (1999).

27) Porsolt R. D., Anton G., Blavet N., Jalfre M., Eur. J. Pharmacol., 47, 379-391 (1978).

28) Onodera K., Kisara K., Ogura Y., Arch. Int. Pharmacodyn. Ther., 240, 220-227 (1979).

29) Onodera K., Arch. Int. Pharmacodyn. Ther., 285, 263-276 (1987)

30) Hakim A. M., Ann. Neurol., 16, 673-679 (1984).

31) Langlais P. J., Mandel R. J., Mair R. G., Behav. Brain Res., 48, 177-185 (1992).

32) Langlais P. J., Savage L. M., Behav. Brain Res., 68, 75-89 (1995).
33) Mair R. G., Otto T. A., Knoth R. L., Rabchenuk S. A., Langlais P. J., Behav. Neurosci., 105, 351-359 (1991).

34) Mair R. G., Knoth R. L., Rabchenuk S. A., Langlais P. J., Behav. Neurosci., 105, 360-374 (1991).

35) Baddeley A. D., Logie R., Bressi S., Della Sala S., Spinnler H., Q. J. Exp. Psychol., 38A, 603 -618 (1986).

36) Oscar-Berman M., Hunter N., Bonner R. T., Behav. Neurosci., 106, 613-622 (1992).

37) Blokland A., Brain Res. Rev., 21, 285-300 (1996)

38) Yabe T., Toriizuka K., Yamada H., Phytomedicine, 2, 41-46 (1995).

39) Yabe T., Toriizuka K., Yamada H., Phytomedicine, 2, 253-258 (1996).

40) Arendt T., Bruckner M., Bigl V., Marcova L., J. Comp. Neurol., 351, 189-222 (1995) .

41) Pitkin S., Savage L. M., Behav. Brain Res., 119, 167-177 (2001).

42) Suzuki T., Higuchi M., Yabe T., Yamada H., Toriizuka K., Hanawa T., Ikarashi Y., Iwasaki K., Wang Q., Arai H., Sasaki H., Abstracts of papers, 29th Annual Meeting, Society of Neuroscience, Miami, October 1999, Vol. 25, p. 848.

43) Nakagawasai O., Yamadera F., Iwasaki K., Arai H., Taniguchi R., Tan-No K., Sasaki H., Tadano T., Neuroscience, 125, 233-241 (2004)

44) Bergeron C., Kish S. J., J. Neurochem., 61, 2007-2014 (1993).

45) Lindsay J. G., Bettendorf L., Rice J., Kish S. J., Ann. Neurol., 39, 593-598 (1996).

46) Savage L. M., Chang Q., Gold P. E., Learn. Mem., 10, 242-246 (2003).

47) Fadda F., Cocco S., Stancampiano R., Neuroreport, 11, 2265-2269 (2000).

48) Wang Q., Iwasaki K., Suzuki T., Arai H., Ikarashi Y., Yabe T., Toriizuka K., Hanawa T., Yamada H., Sasaki H., Phytomedicine, 7, 253-258 (2000).

49) Yabe T., Iizuka S., Komatsu Y., Yamada H., Phytomedicine, 4, 199-205 (1997). 